\title{
La criminologie littéraire $\&$ le récit de sa consécration (La criminologie en poésie et en prose)
}

\section{Genèse \\ Sophie COUSINEAU, Université d'Ottawa}

Les " passerelles du dedans au dehors », titre du dossier présenté dans ce numéro, ne s'actualisent pas uniquement par un projet de rencontres littéraires en milieu carcéral et communautaire. En effet, cette mouvance a ses échos au département de criminologie de l'Université d'Ottawa. Bien sûr, il y a des projets de recherche menés par des candidats au doctorat et à la maîtrise qui empruntent une démarche artistique ou qui explorent des initiatives artistiques en prison. Ces derniers s'inscrivent dans la Chaire de recherche $L a$ prison dans la culture, la culture dans la prison ${ }^{1}$ dont Sylvie Frigon en est la détentrice. Cette Chaire multiplie les initiatives « du dedans au dehors ». Plus encore, cette mouvance se traduit par des méthodes pédagogiques. Dans le cadre du séminaire de maîtrise CRM6780A : Genre, enfermement et créativité, Sylvie Frigon mobilise divers médiums artistiques pour aborder cet enjeu autrement. En 2010, les étudiants participaient d'abord à des ateliers de slam-poésie dirigés par Louise Poirier et ensuite à un spectacle-partage avec les femmes de la Société Élizabeth Fry de Gatineau. En 2012, les étudiantes dansaient sous la tutelle de Claire Jenny, chorégraphiant le cloisonnement du corps dans les cellules de l'ancienne prison d'Ottawa-Carleton.

En 2013, Michèle Vinet a animé des ateliers en création littéraire pour aborder la prison par le filtre de la prose et de la poésie. Procédant à la manière d'un entonnoir, elle a d'abord présenté des exercices portant sur des thèmes généraux et visant à inspirer les actes d'écriture. Les correspondances visaient l'élaboration de métaphores et les déclencheurs, un texte orienté. D'autres mini-récits ont été produits à partir d'interrogations évaluant une image quelconque. Ces mêmes exercices ont ensuite été appliqués à la question de l'enfermement. Après trois séances, les étudiantes avaient composé une dizaine de textes individuels et collectifs. Au cours de la quatrième séance, il y a eu une mise en lecture de ces textes par les étudiantes devant un public. De plus, l'évaluation finale a porté sur la rédaction d'un récit autour de l'enfermement. Voici le résultat de cette expérience collaborative.

\section{Écrits généraux}

\section{Sans titre}

(Véronique PROULX, Kristine PHILIPPE, Sophie COUSINEAU et Michèle VINET)

Un livre

est une vie

un parcours

tortueux

et scintillant

au bout

du chemin

de solitude 
Les vagues sont

des danses

rythmées de la

tête

échevelée de

ma nature

sauvage

*

Condamnation

transposition carcérale

écart social

soif de liberté

désir de couleurs

de chaleur campagne

d'odeur-famille

de promenades matins

et d'épanouissement

du jardin intérieur

*

Dans l'attente

une maternité verrouillée

dépouillée du rouge amour

de la douceur

de l'innocence

de son enfant

une séparation bourreau

privation barbare

amputation

charnelle et affective

Le macadam intérieur (Véronique PROULX)

Un chemin

personnel

aventureux

et soucieux

se perdre

dans l'obscurité

vers l'harmonie

Sans titre (Véronique PROULX)

Les étoiles

sont des images

inaccessibles

perdues 
dans l'atmosphère

des rêves

qui flottent

vers une réalité

qui nous

rendra visite

après des années

lumière

Sans titre (Sophie COUSINEAU)

Un regard sur le passé

Temps de pause

Repli sur soi

Cœur tendre

Refoulement superficiel

Mosaïque

Des interdits de société

Amitié (Kristine PHILIPPE)

L'amitié est

une perle rare

qui brille

au fil des jours

sous les feux d'un amour

résistant aux détours

d'une vie compliquée

Sans titre (Véronique PROULX)

Le café noir

est la source

de mes études

l'oxygène

et l'énergie

d'un goût

amer.

Un livre (Sophie COUSINEAU)

Est une banque de mots

De représentations

De fiction

Et ses fluctuations

De récits hypothéqués

De souvenirs en épargne

D'adjectifs en ristourne

De personnages qui capitalisent

Le crédit, le chèque ou le comptant 
D'actions et de placements

En montagnes russes

Un crash

Un boom

Une perte

Un revenu

Un dénouement à somme nulle

Une mère et son enfant (Kristine PHILIPPE)

Un amour nouveau

Une joie sereine

Un chant apaisant

Au lever du jour

Au réveil de l'innocence

Un environnement de douceur

Dans une intimité sans pareil

La dame lit un livre (Véronique PROULX)

Lorsque j'étais enfant

ma mère me chantait son vécu, là où elle a rencontré mon père, mon histoire préférée

chassant les mauvais rêves

\section{Les écrits autour de l'enfermement}

Désir (Kristine PHILIPPE)

Un visage froid

Au teint pâle

Aux yeux trop grands

Aux joues creusées

Aux cheveux emmêlés

Un visage absent

Cache l'existence

D'une blessure profonde

D'un amour recherché

D'un contact désiré

Rêvé

Malgré cette prison

Cette cellule

Macadam intérieur (Sophie COUSINEAU)

Mec à dames

Emmuré un moment

Partage d'une affection carcérante

Retrouvailles obstacles 
Au bout des doigts

Amour contorsionné

Aux exigences du parloir

Étreintes balisées par des contacts

En-cimentés

Réciprocité à moitié

Minutée et surveillée

Bouée de sauvetage épidermique

Mains crampons

Soif de peau contrainte à boire la mer

Intimité publique

Impudique

Oasis de baisers métalliques

Douceur amère d'un entre-soi

Entre deux cœurs barbelés

Sans titre (Véronique PROULX)

Une femme crispée

dans sa tristesse

un trou

de désespoir

noyé

noirceur

moments de souvenir

et de rêves

de son fils

porteur de bonté

pour survivre

à ce cauchemar

Fer (Kristine PHILIPPE)

Solitude

Souffrance

Tête lourde

Étourderies

Brouillard

Si seulement

j'étais un oiseau

Des ailes.

pour m'envoler

pour m'échapper

Loin.

Ailleurs. 
Impossible.

Ces barreaux...

Sales

Froids

Sonores.

La chute (Sophie COUSINEAU)

Les talons hauts en société

Les pieds nus en prison

Féminité menottée

Dégradée

Perte d'équilibre

Envolée référentielle

Nom pris en otage

Troc de liberté

Qui dépouille

Rôles et statuts

De plomb ou de sel

La mère est amère

L'épouse a les blues

Féminité incarcérée

Parade d'un corps-uniforme

Violé par le regard obsédé

Et ses impératifs de sécurité

Le récipient de maternité

Habitacle de plaisirs-abus

Trafics débusqués par la fouille

Corps-moquette

Aux mocassins dépareillés

La marche (Véronique PROULX)

file indienne

ciel déchiré

femmes

structurées

et ordonnées

vers

une pendaison

de liberté

une détention

barbelée

L'espoir en baluchon (Sophie COUSINEAU)

Pierre angulaire

Clef de survie

À l'incarcération 
Involontaire

Création d'un écart

Et ses rations

Impuissance

Détresse

Un cri trop grand

Noué dans la gorge

Captif de poumons-prison

Non-coupable

Placardé sur le visage

Injustice

Tatouée sur le corps-pantin

Étreinte souffrance

Entre la chair et le fer

Un regard suppliant

Adressé à liberté

Qui en retour

Nous fait la grimace

Nous tourne le dos

Nous dit : à la prochaine

Sans voix (Kristine PHILIPPE)

Le cubicule

Trop petit pour y vivre

Trop restreint

Semblable à une cage animale

Lieu inconcevable

Pour l'humain

Voilà ce qu'est l'isolement

Sa victime aux cent pas circulaires

Au dos recroquevillé

Sans voix

Sans espoir

D'un jour se faire entendre

Si seulement

On m'avait écouté

Si seulement

On m'avait permis de m'expliquer

\section{Purger une peine (Véronique PROULX)}

S'éteindre de nos rêves

pour se figer dans un présent de béton et de barbelés monotones

la fenêtre extérieure d'un jaune secours

me permet de m'évader de cette

solitude éternelle 
La fermeture de la cellule d'un animal en captivité, verrouillé du monde pour atténuer mes plaisirs de vie qui ressurgiront un jour comme des fleurs dans la neige.

\author{
Lame contre larme (Sophie COUSINEAU) \\ Sonner l'alarme \\ Du for intérieur \\ Lacérer la chair \\ Déligoter les émotions \\ Sectionner un ligament \\ Paroxysme \\ Dé-tension \\ Devenue zen \\ Rouge clarté \\ Psyché en évasion \\ Corps morcelé \\ Échoué sur la plage-horaire \\ Hyper-structurée \\ D'un milieu bicolore \\ Inodore \\ De l'enfer \\ Du moment
}

Château et dragons (Kristine PHILIPPE)

Quand j'étais petite, ma mère me racontait des histoires pour justifier les raisons de ses départs. Une tristesse profonde m'envahissait toujours lorsque je m'apercevais qu'une nouvelle aventure m'attendait. Mais, à la fois, mon esprit s'éprenait d'une curiosité et je ne pouvais m'empêcher de prendre place sur le vieux tapis orangé, rongé par l'humidité et les années, de croiser les jambes puis de tendre l'oreillle attentivement, le regard brillant des futurs exploits de maman. Ces aventures me justifiaient les absences de ma mère : ses départs alors que nous venions enfin de nous retrouver. J'étais petite à l'époque. Les princes et les châteaux, j'y croyais. À quoi aurait servi la vie si on ne s'éprenait pas d'un beau prince? Ou, pire encore, si on ne trouvait jamais son château avec ses cheveaux blancs, ses immenses cuisines, ses escaliers en colimaçon et ses énormes fenêtres laissant pénétrer le soleil ?

Mon héroïne me bordait, puis s'étendait dans mon minuscule lit avec moi. Je lui demandais alors, une seconde fois, de me raconter dans les moindres détails ces aventures merveilleuses qui l'attendaient. Elle reprenait son récit, un sourire aux lèvres. Parfois, une larme coulait sur sa joue et elle s'empressait de la sécher. Je ne comprenais pas: pourquoi pleurait-elle si elle était heureuse ? Ne souriait-on pas quand on était heureux ? Le matin suivant, lorsque j'ouvrais les yeux, maman avait quitté. Je savais que je ne pouvais prédire quand j'allais la revoir tout comme elle ne pouvait me le dire. Maman m'avait tout expliqué : elle quittait pour me protéger des méchants dragons. Elle se rendait au château d'un roi très puissant, terriblement loin de notre maison. Il y a plusieurs années, elle avait signé une entente avec lui. Elle lui avait promis d'assurer la 
sécurité des enfants de ce monde. Lorsqu'il lui demandait de se présenter à son château, elle n'avait pas d'autre choix que de s'y rendre et de respecter son entente.

Maman m'avait décrit le château de ce roi à plusieurs reprises. Il était un peu différent des autres. Une forteresse de pierres grises s'étendait le long de ses frontières, elle s'élevait si haut dans le ciel qu'elle pouvait toucher les nuages. Le château était divisé en plusieurs petites tours dans lesquelles les mamans veillaient à trouver des solutions pour l'avenir du pays de ce roi. Un jour, elle m'avait expliqué qu'entre ces murs énormes, elle combattait des dragons de couleurs orange, mauves et jaunes, aux longues dents pointues et aiguisées.

Des histoires comme celles-ci ont sillonné les rêves de mon enfance. J'ai longtemps cru à ces affrontements contre les méchants dragons et à ces châteaux majestueux. Mais aujourd'hui, le visage creusé par l'expérience, j'ouvre les yeux sous le poids opressant de cette obscurité étourdissante et de cette froideur contaminante, léchant graduellement mon corps puis mon âme, et je comprends. Je comprends que les combats contre ces bêtes maléfiques sont la démence qui hante mon esprit, derrière cette forteresse trop longtemps écrasante, outil de la suppression de ma liberté.

Aux histoires de ma mère, j'ai longtemps cru. Comme mes enfants y croiront à leur tour.

${ }^{1}$ Pour de plus amples détails, consultez l'hyper-lien suivant :

http://sciencessociales.uottawa.ca/recherche/chaires-recherche-faculte 